\title{
Roedores inventariados em hospital veterinário e fragmento de mata nativa da Zona da Mata de Minas Gerais, Brasil: caracterização populacional e infecção por Leptospira sp.
}

\author{
Rodentes captured in a veterinary hospital and a fragment of atlantic forest in Zona da Mata, Minas Gerais \\ state, Brazil: populacional characterization and Leptospira sp. infection
}

\author{
Paula Dias Bevilacqua ${ }^{1}$ Rose Ferraz Carmo ${ }^{2}$ João Carlos Pereira da Silva ${ }^{3}$ \\ Gisele Mendes Lessa Del Giudice ${ }^{4}$
}

RESUMO

Os roedores podem transmitir enfermidades para o ser humano e animais, além de provocar diversos prejuízos econômicos. Objetivou-se estudar a população de roedores existente em um hospital veterinário universitário e em um fragmento de mata atlântica nativa, ambos os locais localizados na Zona da Mata de Minas Gerais, Brasil. No hospital veterinário, foram analisadas as variáveis espécies, sexo, período reprodutivo, sazonalidade, presença de Leptospira $s p$. em fragmento renal e anticorpos anti-leptospira em amostras de soro; os animais silvestre foram analisados segundo a presença de Leptospira sp. em fragmento renal. No hospital veterinário, foram capturados 72 animais, 22 (30,6\%) caracterizados segundo a espécie Rattus rattus, sendo a maioria $(66,7 \%)$ capturada no setor de pequenos animais. Não foram encontradas diferenças estatisticamente significativas entre machos e fêmeas em período reprodutivo $\left.\chi^{2}=1,85 ; p=0,174 ; \alpha=0,05\right)$. Houve captura de animais em todos os meses do ano, sendo que os meses janeiro (12,5\%), fevereiro $(15,2 \%)$, maio $(15,2 \%)$ e julho $(16,7 \%)$ apresentaram as maiores proporções e agosto (2,8\%), setembro $(4,2 \%)$ e outubro $(1,4 \%)$, as menores. Não foram encontrados animais positivos para leptospirose através do teste sorológico da microaglutinação e da pesquisa histopatológica. Os gêneros capturados no fragmento de mata foram: 16 (50\%) Oligoryzomis sp, 11 (34,3\%) Akodon sp, 3 (9,3\%) Oximicterus sp, 1(3,2\%) Bolomys sp, $1(3,2 \%)$ Oryzomis sp. Também nesses não se encontrou resultado positivo na pesquisa histopatológica. A presença de Rattus rattus no hospital veterinário é explicada pela existência de locais que servem de esconderijo e de alimento disponível em quantidade e qualidade adequada a esta espécie. Estas condições predispõem à manutenção de roedores durante todo o ano e de machos $e$ fêmeas aptos para a reprodução. A ausência de roedores com resultados positivos para leptospirose não os exime como potenciais reservatórios desta enfermidade, uma vez que o hospital veterinário, por se tratar de ambiente hospitalar, pode eventualmente abrigar espécies domésticas para tratamento que estejam eliminando a Leptospira sp pela urina. As espécies silvestres capturadas, aparentemente, não representam reservatórios da leptospirose.

Palavras-chave: roedores, dinâmica populacional, leptospirose, hospital veterinário.

\section{ABSTRACT}

Rodents can transmit several diseasesto to human and other animals and cause economic damages. This study looked at rodent population in a veterinary hospital concerning the followings aspects: rodent specie, sex, reproductive period, seasonality and research for Leptospira sp. In addition, rodents from an Atlantic forest fragment were captured and examined for Leptospira sp. Infection. Both the hospital and the forest were located at Zona da Mata region, Minas Gerais state, Brazil. 72 animals were captured at the veterinary hospital, 22 (30.6\%) had been characterised according to Rattus rattus species and most of them $(66.7 \%)$ were captured in small animals sector. There were no significant differences between males and females in reproductive period $\left(\chi^{2}=1.85 ; p=0.174 ; \alpha=0.05\right)$. Rodents were captured all over the year. The highest percentages were observed in January (12.5\%), February (15.2\%), May (15.2\%) and July (16.7\%), and the lowest in August (2.8\%), September (4.2\%) and October (1.4\%). There was no positive identification of animals for Leptospira sp., by microagglutination and histopathological exams. The animals from the forest were identified as wild rodents. $16(50 \%)$ Oligoryzomis sp, 11 (34.3\%) Akodon sp, 3 (9.3\%) Oximicterus sp, 1(3.2\%) Bolomys sp, 1 (3.2\%) Oryzomis sp. There weren't either any findings of positive animals by histopathological exam. The presence of Rattus rattus in the veterinary hospital could be explained by the existence of appropriate places for hiding and food availability, in amount and quality suitable to this species. These conditions allow for the maintenance of rodents during all over the year, and of males and

${ }^{1}$ Médico veterinário, Doutor em Epidemiologia , Professor adjunto, Departamento de Veterinária (DV), Universidade Federal de

Viçosa (UFV), Viçosa-MG. E-mail: paula@ufv.br. Autor para correspondência.

${ }^{2}$ Médico veterinário, estudante de Pós-graduação, DV, UFV.

${ }^{3}$ Médico veterinário, Doutor em Patologia, Professor Adjunto, DV, UFV.

${ }^{4}$ Licenciado em Ciências Biológicas, Mestre em Ciências Paleontologicas, Professor Assistente do Departamento de Biologia Animal, UFV. 
females ready for reproduction. At the hospital, the absence of positive results for Leptospira sp. do not exclude the potential of the rodents as reservoirs of such pathogen, since a veterinary hospital can always shelter domestic species for treatment that could be eliminating the Leptospira sp. The captured wild species apparently do not represent reservoirs of Leptospira sp.

Key words: rodents, population dynamics, leptospirosis, veterinary hospital.

\section{INTRODUÇÃO}

Os roedores têm merecido a atenção de pesquisas da área da saúde humana e produção animal, basicamente, em função de dois grandes aspectos: (i) são reservatórios de importantes doenças, inclusive com significado zoonótico, (ii) são responsabilizados por importantes perdas econômicas exemplificado pelo fato de consumirem grandes parcelas de alimentos armazenados.

O ambiente hospitalar não deve representar risco à saúde dos pacientes que eventualmente permaneçam em suas dependências, sendo que o mesmo significado deve ser considerado para o ambiente de um hospital veterinário. Entretanto, em hospitais veterinários, pelas peculiaridades dos pacientes internados, pode haver acúmulo de resíduos que são excelentes fontes de alimentos para roedores, como restos de alimentos fornecidos aos animais e material utilizado como cama para os mesmos. Essas características favorecem a presença de roedores, sendo que, em função do tipo de ambiente e dos alimentos disponíveis, pode haver predomínio de diferentes espécies.

O conhecimento da(s) espécie(s) de roedores que infestam áreas específicas e a dinâmica dessa população se torna importante à medida que as ações de controle, sejam de antiratização ou desratização, devem ser planejadas e executadas também em função da espécie que se quer controlar. Esse conhecimento é um dos pressupostos do que atualmente é entendido como manejo integrado de roedores.

Com a finalidade de subsidiar um programa de controle de roedores em ambiente hospitalar, foi realizado inventário das espécies existentes nas dependências de um hospital veterinário universitário. A partir dos animais capturados durante o inventário, foi investigada a existência de anticorpos anti-leptospiras e a presença deste agente em fragmentos de tecido renal, com o intuito de avaliar o papel das espécies de roedores identificadas como reservatórios de Leptospira sp.

Paralelamente a estas atividades, foi realizada a pesquisa de Leptospira sp. em tecido renal de espécies silvestres de roedores. Esses animais foram capturados em fragmento de mata nativa localizada na Zona da Mata de Minas Gerais, Brasil permitindo avaliar o papel desempenhado por espécies silvestres como reservatórios da leptospirose.

\section{MATERIALE MÉTODOS}

\section{Captura dos animais}

O inventário da população de roedores no hospital veterinário universitário foi conduzido com a utilização de armadilhas do tipo "gancho" e "sherman" iscadas com uma mistura de frutas (banana), farelo de milho e óleo de fígado de bacalhau. As armadilhas eram colocadas diariamente, durante sete dias consecutivos, no período noturno, e recolhidas no dia seguinte. Este processo foi repetido mensalmente entre fevereiro de 2002 a janeiro de 2003.

Para a realização do inventário foram priorizadas as áreas onde eram verificadas as maiores ocorrências de roedores (Setor de Clínica de Grande Animais e de Pequenos Animais) e áreas externas limítrofes com a mata que circunda as porções Norte e Leste do hospital veterinário. Inicialmente, foram utilizadas 40 armadilhas, sendo 17 do tipo "sherman" e 23 do tipo "gancho". Ao final do inventário, a partir de dezembro de 2002, por motivos de desaparecimento ou não funcionamento, estavam sendo utilizadas 30 armadilhas, das quais 13 eram do tipo "sherman" e 17 do tipo "gancho".

No momento da coleta dos animais capturados, estes eram identificados e avaliados quanto a estarem ou não em período reprodutivo. Nos animais machos, a existência de bolsa escrotal com testículos descidos era indicativo de animal apto para a reprodução. Esta condição foi caracterizada nas fêmeas quando estas apresentavam vagina perfurada. Após esta avaliação, os animais capturados eram sacrificados com éter, sendo logo após submetidos à necrópsia para coleta de sangue e fragmentos de tecido renal.

Os animais silvestres utilizados nesse trabalho foram capturados por pesquisadores do Departamento de Biologia Animal da UFV, autorizados por órgão competente para a coleta. $\mathrm{O}$ período de captura compreendeu os meses de novembro de 2002 a fevereiro de 2003, sendo utilizadas armadilhas do tipo "sherman" e baldes plásticos enterrados à altura do solo. A isca utilizada foi constituída de banana e fubá de milho. Os animais capturados eram sacrificados com éter e submetidos à necropsia para coleta de fragmento renal. Não foi possível realizar a coleta de sangue 
para pesquisa de anticorpos anti-leptospira pela técnica da microaglutinação devido ao reduzido tamanho das espécies capturadas.

Processamento do sangue e dos fragmentos de tecido renal

O sangue coletado com seringas descartáveis era transferido para tubos de ensaio e deixado em repouso, à temperatura ambiente para separação do soro. Logo após, o soro era separado em tubos "eppendorf", identificado e armazenado à $-20^{\circ} \mathrm{C}$, para posterior análise sorológica. Os fragmentos de rim obtidos durante a necrópsia eram transferidos para frascos contendo formalina $40 \%$, identificados e armazenados para posterior análise histopatológica.

Taxidermização dos animais

Os animais capturados foram taxidermizados segundo técnica descrita por MOOJEN (1943). Para se proceder à classificação das espécies de roedores, foram utilizados padrões de medidas corporais associados a características externas como a presença de membrana interdigital e calos palmares, plantares dentre outras, conforme EMMONS \& FEER (1990).

\section{Análises laboratoriais}

As amostras de soro coletadas dos animais capturados no hospital veterinário foram testadas pela técnica da microaglutinação rápida (RYU, 1970) utilizando-se antígenos vivos de 10 sorovariedades de Leptospira interrogans: autumnalis, bratislava, canicola, castellonis, grippotyphosa, hardjo, icterohaemorrhagiae, pyrogenes, tarassovi e wolffi. Os soros foram diluídos na razão 1/50 e considerados positivos os que apresentassem reação de aglutinação em observação em microscópio de campo escuro.

O tecido renal coletado foi utilizado para a realização da técnica histopatológica de impregnação pela prata para visualização da bactéria nos túbulos renais, segundo protocolo descrito em LUNA (1968).

\section{Análise dos dados}

Os dados foram analisados segundo técnicas da estatística descritiva, através de tabelas de distribuição de freqüência. Para avaliação da existência de associação entre variáveis, local de captura de animais, tipo de armadilha utilizada, sexo e período reprodutivo dos animais foi utilizada a técnica do qui-quadrado, adotando-se o nível de significância de 95\%, conforme FLEISS (1981). A verificação da existência de sazonalidade do número de animais capturados foi realizada através da análise da distribuição mensal dos resultados. As análises estatísticas foram desenvolvidas no programa EpiInfo, versão 6.0 (WHO, 1997).

\section{RESULTADOSEDISCUSSÃO}

Caracterização das espécies de roedores capturados nas dependências do hospital veterinário

Foram capturados 72 animais, sendo que 22 $(30,6 \%)$ roedores foram taxidermizados e caracterizados como Rattus rattus. O restante, 50 animais $(69,4 \%)$, não passou pelo processo de taxidermia devido a óbito ou perda do material durante o processamento. Entretanto como só foi encontrada uma única espécie é provável que estes animais também pertençam à espécie Rattus rattus. $\mathrm{O}$ encontro de animais apenas dessa espécie nas dependências do hospital veterinário, indica a existência de condições favoráveis à proteção e à reprodução destes animais, tais como ausência de espécies predadoras como Rattus norvegicus e disponibilidade de alimentos em grãos como ração utilizada na alimentação de bovinos e eqüinos e restos de alimentos utilizados na alimentação de cães e gatos internados no hospital. A existência da espécie Rattus rattus neste ambiente é bastante condizente com a característica sinantrópica deste roedor muito bem adaptado ao ambiente peri-domiciliar e domiciliar (FORATTINI, 1992; NAGY, 1993; BRASIL, 2002b).

Apesar do hospital veterinário estar localizado próximo a um fragmento de mata, não foram capturados roedores silvestres em suas dependências, mesmo naquelas armadilhas colocadas em áreas próximas aos limites do hospital com a mata.

Local de captura e armadilha utilizada

A distribuição dos roedores capturados segundo o local revela que a maioria dos roedores $(66,7 \%)$ foi capturada no setor de pequenos animais (cães e gatos) integrante das dependências do hospital veterinário, o que pode estar associado à prática de se deixar por períodos prolongados de tempo os restos de alimentos utilizados na alimentação dos animais internados, funcionando, assim, como atração para os roedores. Considerando apenas as capturas com informações referentes ao lugar (53), encontramos diferença estatisticamente significativa na distribuição $\left(\chi^{2}=63,44\right.$; $\mathrm{p}<0,00001 ; \alpha=0,05)$ indicando maiores proporções de roedores capturados no setor definido no parágrafo anterior, mais especificamente $43,4 \%$ nas baias de internação de cães de maior porte, 26,4\% nas baias de internação de cães de menor porte e gatos e $20,8 \%$ no solário de cães e gatos (Tabela 1).

Durante o período de captura, 40,3\% dos roedores foram capturados em armadilhas "sherman" 
Tabela 1 - Número e distribuição proporcional de animais capturados segundo o local de captura, hospital veterinário, fevereiro de 2002 a janeiro de 2003.

\begin{tabular}{lcc}
\hline Local & Animais capturados & Porcentagem $(\%)$ \\
\hline Baias de internação de cães de maior porte & 22 & 30,6 \\
Baias de internação de cães de menor porte e gatos & 14 & 19,4 \\
Solário de cães e gatos & 11 & 15,3 \\
Canais de esgotamento sanitário & 3 & 4,2 \\
Corredor das baias de internação de cães de maior porte & 1 & 1,4 \\
Setor de anatomia & 1 & 1,4 \\
Baias de internação de animais de médio porte & 1 & 1,4 \\
Sem informação & 19 & 26,3 \\
TOTAL & 72 & 100,00 \\
\hline
\end{tabular}

e 38,9\% em armadilhas "gancho". Ambos os tipos usados foram igualmente eficazes na captura dos roedores nas dependências do hospital veterinário, não evidenciando diferenças entre os tipos de armadilhas utilizados $\left(\chi^{2}=0,04 ; p=0,851 ; \alpha=0,05\right)$. A adequação das armadilhas deve-se ao fato da população de roedores existente na área trabalhada, conforme pode ser comprovado com este estudo, ser constituída basicamente de Rattus rattus, o qual apresenta tamanho e constituição física compatível com estes tipos de armadilhas.

Caracterização dos roedores capturados segundo o sexo e o período reprodutivo

Dentre os 72 roedores capturados, pode-se caracterizar quanto ao sexo e o período reprodutivo 61 animais $(84,7 \%)$. Com relação ao sexo, encontramos $47,5 \%$ de machos e $52,5 \%$ de fêmeas, não havendo diferença estatisticamente significativa entre estas proporções $\left(\chi^{2}=0,13 ; p=0,719 ; \alpha=0,05\right)$.

A caracterização do período reprodutivo revelou praticamente a mesma quantidade de machos aptos (15) e não aptos (14) para a reprodução, sendo que um animal não pode ser caracterizado. Do total de fêmeas capturadas, 68,8\% estavam aptas para a reprodução e 31,2\% não aptas. A distribuição de machos e fêmeas aptos ou não para a reprodução também não apresentou diferença estatisticamente significativa $\left(\chi^{2}=1,85 ; p=0,174 ; \alpha=0,05\right)$, indicando que os roedores encontram condições favoráveis para a reprodução durante $\mathrm{o}$ ano inteiro.

Caracterização dos roedores capturados segundo o mês A figura 1 mostra a distribuição mensal dos animais capturados. Os meses de janeiro, fevereiro, maio e julho apresentaram, proporcionalmente, maior número de animais capturados. De forma inversa, os meses de agosto setembro e outubro foram os que apresentaram as menores proporções de roedores. $\mathrm{O}$ fato de que esses meses se caracterizaram por intensas precipitações atmosféricas e baixas temperaturas, pode ter prejudicado o sucesso das capturas realizadas.

Caracterização dos roedores capturados segundo o resultado da prova de aglutinação microscópica e do exame histopatológico

Dentre os animais capturados nas dependências do hospital veterinário, não se encontraram animais com sorologia positiva para Leptospira sp. nem fragmentos renais onde a bactéria pudesse ser visualizada. Estes resultados estão de acordo com os dados de literatura que imputam muito mais ao Rattus norvegicus a responsabilidade pela transmissão da leptospirose aos seres humanos (LOMAR et al., 1997;BRASIL, 2002a).

Entretanto, não se podem descartar a possibilidade desta espécie de roedor poder se contaminar com a Leptospira sp. e vir a funcionar como reservatório, uma vez que o ambiente no qual estes animais circulam constitui área hospitalare pode receber em suas instalações animais de estimação ou de produção doentes ou portadores, em fase de eliminação renal da bactéria.

\section{Animais capturados no fragmento de mata nativa}

Foi capturado um total de 32 roedores silvestres, dos quais $16(50 \%)$ pertenciam ao gênero Oligoryzomis sp, 11 (34,3\%) pertenciam ao gênero Akodon sp, 3 (9,3\% pertenciamao gênero $O$ ximicterus sp, 1 (3,2\%) foi identificado como Bolomys spe 1 (3,2\%) como Oryzomis sp.

Do total de animais capturados, $23(71,9 \%)$ eram machose 9(28,1\%), fêmeas. A distribuiçãomensal dos animais capturados foi a seguinte: novembro de 2002 - 5 (15,6\%), dezembrode 2002 - 15(46,9\%), janeiro de 2003 - 11 (34,3\%), fevereirode $2003-1(3,2 \%)$.

Com relação aos animais silvestres capturados, destaca-se o fato de não se ter verificado a existência de animais positivos ao exame histopatológico para Leptospira sp. Estes resultados podem indicar a pequena ou nenhuma importância de roedores silvestres, 


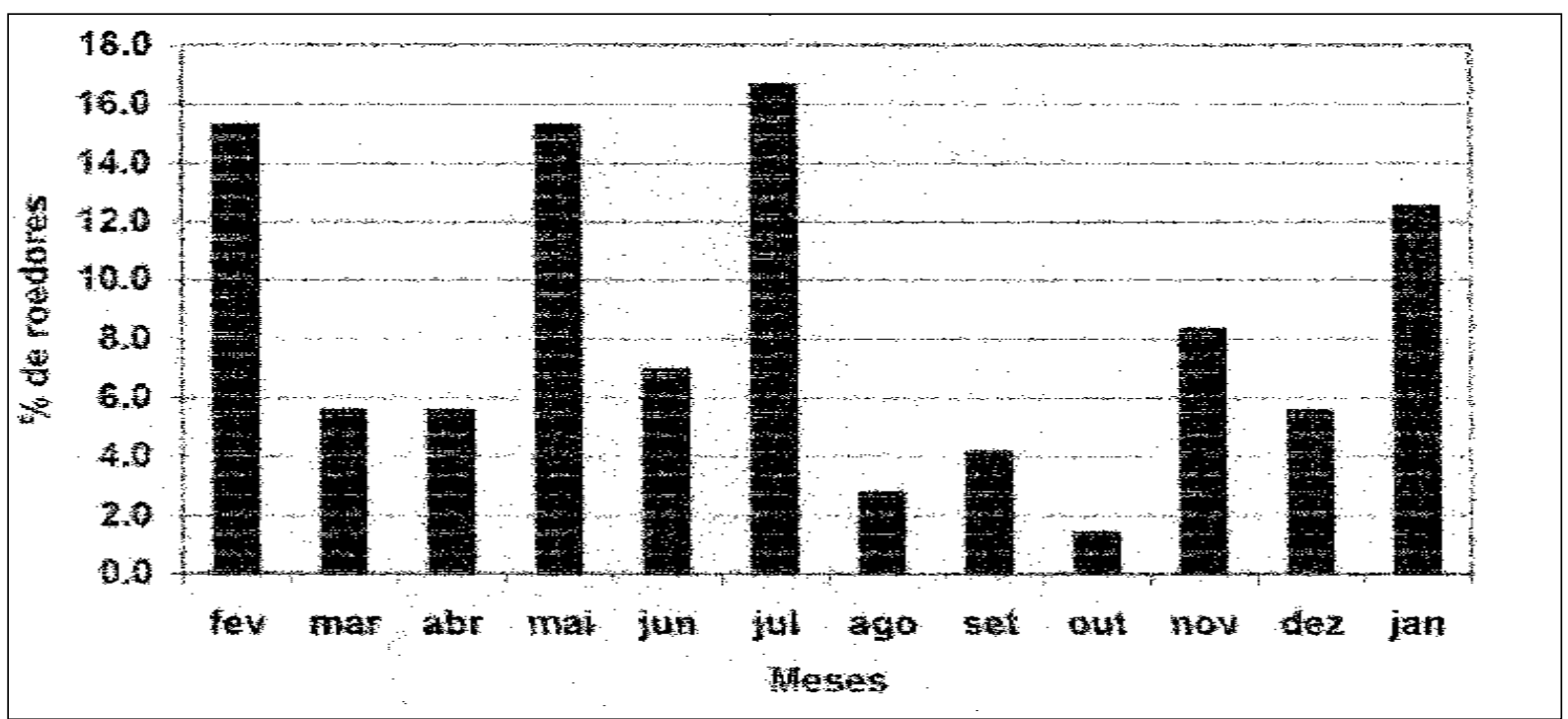

Figura1 - Distribuição mensal dos roedores capturados, hospital veterinário, fevereiro de 2002 a janeiro de 2003.

notadamente quando habitando áreas protegidas, livres de explorações animais, na manutenção da leptospirose no ambiente, não descartando, entretanto, a possibilidade de espécies silvestres participarem como reservatórios da leptospirose, apesar de usualmente não serem relatadas na literatura como tal.

\section{CONCLUSÕES}

O inventário de roedores realizado no hospital veterinário revelou a presença de uma única espécie sinantrópica, Rattus rattus, não tendo sido verificada diferença entre os animais com relação ao sexo e ao período reprodutivo. $\mathrm{O}$ ambiente mais infestado por roedores foi o setor de pequenos animais

Com relação aos aspectos sanitários, os exemplares capturados no hospital veterinário universitário não apresentaram indícios de infecção anterior ou recente por Leptospira sp., o que não significa que menor atenção seja dispensada ao controle dessa espécie, por, aparentemente, não serem reservatórios desse agente na área em questão. Os gêneros de roedores silvestres capturados também não foram identificados como portadores de Leptospira sp. provavelmente por terem sido capturados em local de mata protegida e livre de explorações animais que implique a presença de animais portadores e eliminando o agente, funcionando, assim, como fonte de infecção para as espécies silvestres.

\section{AGRADECIMENTOS}

Esse trabalho contou com apoio financeiro da Fundação de Amparo à Pesquisa do Estado de Minas Gerais (FAPEMIG).

\section{REFERÊNCIAS BIBLIOGRÁFICAS}

BRASIL. Fundação Nacional de Saúde. Guia de vigilância epidemiológica. 5.ed. Brasília : FUNASA, 2002a. 842p.

BRASIL. Fundação Nacional de Saúde. Manual de controle de roedores. Brasília : FUNASA, 2002b. 132p.

EMMONS, L.; FEER, F. Neotropical rainforest mammals. Chicago : The University of Chicago, 1990. 281p.

FLEISS, J.L. Statistical methods for rates and proportions. New York : John Wiley \& Sons, 1981. 321p.

FORATTINI, O.P. Ecologia epidemiologia e sociedade. São Paulo: Edusp, 1992. 529p.

LOMAR, A.V. et al. Leptospiroses. VERONESI, R.; FOCACCIA, R. (Ed.). Veronesi: tratado de infectologia. São Paulo : Atheneu, 1997. p.987-1003.

LUNA, L.C. (ed.) Manual of histologic staining methods of the Armed Forces Institute of Pathology. 3.ed. New York : Mc Graw, 1968. 258p.

MOOJEN, J. Captura e preparação de pequenos mamíferos para coleções de estudo. Rio de Janeiro : Imprensa Nacional, 1943. 98p.

NAGY, T. Normas operacionais de centros de controle de zoonoses: Procedimentos para o controle de roedores. Brasília : Fundação Nacional de Saúde, 1993. 80p.

RYU, E. Rapid microscopic agglutination test for Leptospira without non-specific reaction. Bulletin of Oficina Internacional de Epizootias, v.73, p.49-59, 1970 .

WORLD HEALTH ORGANIZATION. World processing, database and statistics e program for public health (EpiInfo). Versão 6.04b. Genebra : WHO, 1997. 